\title{
Foreword
}

\section{Geriatric Anesthesia: Ensuring the Best Perioperative Care for Older Adults}

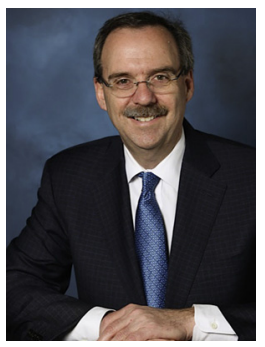

Lee A. Fleisher, MD Consulting Editor

With advances in care of chronic diseases and cancer treatments, there are a growing number of individuals who would be considered both older adults and the very old. These individuals are at great risk of cardiopulmonary, neurologic, and other complications after surgery. It is becoming increasingly clear that those at greatest risk are frail and that surgery may not lead to any improvement in their lives. In addition, optimization or preparation of older adults before surgery may be the best approach to ensuring the best outcome after surgery. In this issue of Anesthesiology Clinics, innovations in the optimal care for older adults are discussed.

In order to bring together a state-of-the-art issue, 2 leaders in geriatric anesthesia were chosen. Elizabeth L. Whitlock, MD, MSc is Assistant Professor of Anesthesia and Perioperative Care at the University of California, San Francisco and focuses on clinical and patient-oriented research. Her research centers on understanding cognitive change over time in the elderly, particularly as it relates to surgery and anesthesia, using population-based data sources, and is funded by the National Institutes of Health. Robert A. Whittington, MD is Professor of Anesthesiology at Columbia University and has a funded basic science laboratory. The Whittington Lab studies the impact of surgery and anesthesia on the progression of Alzheimer disease pathologic condition and its related cognitive decline. Together, they have assembled a 
diverse group of authors and articles, which include novel topics, such as decision making and palliative care.

Lee A. Fleisher, MD Perelman School of Medicine University of Pennsylvania 3400 Spruce Street, Dulles 680

Philadelphia, PA 19104, USA

E-mail address:

Lee.Fleisher@uphs.upenn.edu 